\title{
Explanation of Magnetic Destruction of Superconductor Using Schrodinger Equation in the Energy Space
}

\author{
Einas Mohamed Ahmed Mohamed ${ }^{1,2}$, Mohammed Idriss Ahmed Mohammed ${ }^{3}$, \\ Mubarak Dirar Abd-Alla ${ }^{3}$, Mohamed Yahia Shirgawi Mohammed ${ }^{4}$, Saffa Elsiddig Abd Magid ${ }^{5}$ \\ ${ }^{1}$ Department of Physics \& Mathematics, Faculty of Education, Taif University, Taif, KSA; ${ }^{2}$ University of Gezira, Wad \\ Medani, Sudan; ${ }^{3}$ Department of Physics, Faculty of Science, Sudan University of Science and Technology, Khartoum, \\ Sudan; ${ }^{4}$ Department of Physics, Faculty of Art and Science at AL Muznab, AL Qassim University, AL Muznab, KSA; \\ ${ }^{5}$ Department of Physics, Faculty of Science and Technology, Omdurman Islamic University, Omdurman, Sudan
}

Correspondence to: Mubarak Dirar Abd-Alla, mubarakdirar@gmail.com

Keywords: Superconductivity, Schrodinger Equation, Energy Space, External Magnetic Field, Complex Resistance

Received: February 19, 2020

Accepted: March 22, 2020

Published: March 25, 2020

Copyright $\odot 2020$ by author(s) and Scientific Research Publishing Inc.

This work is licensed under the Creative Commons Attribution International License (CC BY 4.0).

http://creativecommons.org/licenses/by/4.0/

\section{(c) (i) Open Access}

\section{ABSTRACT}

The Wave function of Schrodinger Equation is expressed in terms of time dependent energy eigen function and spatial dependent wave function in the energy space, which gives spatial energy probability. This equation is utilized to find quantum momentum dependent on temperature. This in turn is used to find quantum complex resistance. This expression shows that the superconducting resistance vanishes for temperatures less than a certain critical value. This result conforms to superconductor conventional theory and empirical relations. The application of external magnetic field destroys superconductivity when its strength exceeds a certain critical value. The expression of the relationship between the critical magnetic field and the critical temperature is typical to the conventional one. This is the first time to obtain the conventional relationship for the superconductor's resistance and critical magnetic field in one model in the energy space.

\section{INTRODUCTION}

The history of atomic physics starts and dates from the Plank quantum hypothesis. According to this hypothesis electromagnetic waves behave some times as particles or discrete quanta having energy proportional to the frequency. This means that light and other electromagnetic waves behave as waves and as particles sometimes [1]. Later on Debrogglie proposed that the wave-particle duality characterizes constituents of atoms, atomic and subatomic world in general [2]. This duality represents the cornerstone of the so called quantum mechanics, which describes the behavior of the atomic world [3]. Quantum Mechanical laws describe successfully many physical phenomena like spectra and magnetic resonance [4]. Quantum laws are mainly based on wave particle duality. One of the most widely used ones is Schrodinger equation. 
This equation describes the evolution of the quantum system in terms of the wave function in the coordinate space. When expanding the wave function in terms of the energy eigen function, and substituting it in Schrodinger equation, one gets the evolution of the wave function in the energy space. The Hamiltonian of the quantum system can be expressed as a time operator which gives time dependent energy eigen function. Or it can be expressed as coordinate dependent operator, which gives spatial dependent energy eigen function. The former gives spatially dependent evolution and energy wave function, while the latter gives time dependent evolution and energy eigen function. In spite of these successes quantum mechanics suffer from noticeable setbacks. For example, no quantum gravitational laws are proposed to describe successfully all early universe phenomena besides the behavior of exotic objects like black holes, quasars, pulsars, and neutron stars besides supernova [5]. The behavior of bulk matter sometimes cannot be explained easily by using quantum laws. This includes superconductivity and biophoton behavior $[6,7]$. Some attempts were made to remedy some of these defects. The destroying superconducting state by magnetic field was explained by some models [8]. The pressure effects were also explained by some models [8]. But none of them tries to find the conventional expression for the critical magnetic field and its temperature dependence and at the same time obtains the ordinary empirical vanishing of the superconductor's resistance beyond a certain critical value $[9,10]$.

Thus new alternatives are needed to widen the scope of view for the superconductor's phenomena.

One of these new alternatives was tried by using the energy wave function in the energy space to explain magnetic distraction of superconductor, by derivation of the expression of the critical magnetic field flux density in terms of temperature and critical temperature This is done by expanding Schrodinger wave function in terms of time dependent energy eigen value, which is done in Section 2. Sections 3 and 4 are devoted to discussion and conclusion.

\section{THE ENERGY SPACE SCHRODINGER WAVE FUNCTION MODEL}

The ordinary Schrodinger equation takes the form

$$
i \hbar \frac{\partial \Psi}{\partial t}=-\frac{\hbar^{2}}{2 m} \nabla^{2}+V \Psi
$$

where $\Psi$ stands for the wave function, $V$ represents the potential. Consider that particles behave as a harmonic oscillator affected by electronic crystal potential $V_{0}$ and external magnetic potential $V_{m}$. This means that the total potential $V$ is given by:

$$
V=V_{1}+V_{0}-V_{m}=\frac{1}{2} k x^{2}+V_{0}-V_{m}
$$

where $k$ is the elastic constant and $x$ is the displacement. Where the magnetic potential is assumed to oppose the crystal one. The time dependent wave function takes the form

$$
\begin{gathered}
\Psi=u_{k} \mathrm{e}^{\frac{-i E_{k}}{\hbar} t}=u_{k} \mathrm{e}^{-i w_{k} t} \\
E_{k}=\hbar w_{k}
\end{gathered}
$$

The energy is denoted by $E$ and the angular frequency is denoted by $w$. In contrast to the ordinary conventional energy wave function which is time dependent one assume here spatial dependent energy wave function, by suggesting:

$$
\begin{gathered}
\Psi=\sum_{k} c_{k} u_{k} \\
c_{k}=c_{k}(E, x)
\end{gathered}
$$

The energy eigen function and the energy wavefunction are denoted by $u$ and $c$ respectively. Here the energy eigen function is time dependent in the form: 


$$
u_{k}(t)=u_{k}=A \mathrm{e}^{\frac{-i E_{k}}{\hbar} t}=A \mathrm{e}^{-i w_{k} t}
$$

This is different from spatial dependent energy eigen function which satisfies the relation

$$
\begin{gathered}
\hat{H} u_{n}=\left(-\frac{\hbar^{2}}{2 m} \nabla^{2}+V\right) u_{n}=E_{n} u_{n} \\
u_{n}=u_{n}(x) \\
i \hbar \frac{\partial \Psi}{\partial t}=\sum_{k} E_{k} c_{k} u_{k} \\
\sum_{k} E_{k} c_{k} u_{k}=-\frac{\hbar^{2}}{2 m} \sum_{k} u_{k} \nabla^{2} c_{k}+\sum_{k} V c_{k} u_{k}
\end{gathered}
$$

Multiply both sides by $\bar{u}_{n}$ then use the identity

$$
\int \bar{u}_{n} u_{k} \mathrm{~d} t=\delta_{n k}=|A|^{2} \int \mathrm{e}^{i w_{n} t} \mathrm{e}^{-i w_{k} t} \mathrm{~d} t
$$

Then integrate to get:

$$
\sum_{k} E_{k} C_{k} \int \bar{u}_{n} u_{k} \mathrm{~d} t=-\frac{\hbar^{2}}{2 m} \sum_{k} \int \bar{u}_{n} u_{k} \mathrm{~d} t \nabla^{2} c_{k}+\sum_{k} V_{k} c_{k} \int \bar{u}_{n} u_{k} \mathrm{~d} t
$$

where

$$
V=V_{k}
$$

is time independent

$$
\begin{gathered}
E_{n} C_{n}=-\frac{\hbar^{2}}{2 m} \nabla^{2} c_{n}+V_{n} C_{n} \\
V=V_{n}=V(r)
\end{gathered}
$$

Rearranging yields:

$$
-\frac{\hbar^{2}}{2 m} \nabla^{2} c_{n}=\left(E_{n}-V\right) c_{n}
$$

New one can suggest a solution:

$$
c_{n}=A \mathrm{e}^{i k x}
$$

where:

$$
\nabla^{2} c_{n}=-k^{2} c_{n}
$$

A direct substitution of (15) in (13) gives:

$$
\begin{gathered}
\frac{\hbar^{2} k^{2}}{2 m}=E_{n}-V \\
k=\frac{1}{\hbar} \sqrt{2 m\left(E_{n}-V\right)}
\end{gathered}
$$

Assume now that the total energy is in the form of thermal energy, i.e.:

$$
E_{n}=K T
$$

Assume also that the electrons are affected by a crystal force or repulsive force from surrounding 
electron force gas. In this case, for uniform potential

$$
V=V_{0}
$$

With the aid of Equations (16) and (18), one gets:

$$
K=\frac{1}{\hbar} \sqrt{2 m\left(K T-V_{0}\right)}
$$

The resistance can be expressed as real and imaginary part in the form of real superconducting part $R_{s}$ and imaginary term $R_{i}$, i.e.:

$$
R=R_{r}+i R_{i}=R_{s}+i R_{i}
$$

Bridging the gap between classical and quantum laws through the momentum, one gets:

$$
R=\frac{U}{I}=\frac{m v^{2}}{n e^{2} v A}=\frac{m v}{n e^{2} A}=\frac{\hbar k}{n e^{2} A}
$$

where:

$$
\begin{gathered}
P=m v=\hbar k \\
\frac{V}{e}=\frac{k E}{e}=\frac{1}{2} \frac{m v^{2}}{e}=U
\end{gathered}
$$

Inserting Equation (19) in (21) yields:

$$
R=\frac{\sqrt{2 m(K T-V)}}{n e^{2} A}
$$

$R$ is imaginary when:

$$
K T<V_{0}
$$

Assume now the critical temperature $T_{c}$ to be defined by:

$$
V_{0}=K T_{c}
$$

Thus $R$ is imaginary when:

$$
\begin{gathered}
K T<K T_{c} \\
T<T_{c}
\end{gathered}
$$

where:

$$
R=\frac{\sqrt{2 m k\left(T-T_{c}\right)}}{n e^{2} A}
$$

In this case according to Equations (20) and (27), one gets:

$$
R=R_{i}=\frac{\sqrt{2 m k\left(T_{c}-T\right)}}{n e^{2} A}
$$

In view of Equation (20), the Sc resistance vanishes, i.e.:

$$
R_{s}=0
$$

For all temperatures less than the critical temperature $T_{c}$ defined by Equation (25), i.e.:

$$
T \leq T_{c}
$$

This means that the resistance vanishes for all temperatures beyond the critical temperature $T_{c}$. This result agrees with what is well known fact that the superconductors resistance vanishes beyond a certain 
critical value $[9,10]$.

However let an external magnetic field be applied such that it acts as an attractive force with potential $V_{m}$ other than $V_{0}$ which result from the electron cloud. In this case the total potential is given to be:

$$
V=V_{0}+V_{m}
$$

In view of Equations ((16), (17), (23) and (24)) one gets:

$$
R=\frac{\sqrt{2 m\left(K T-K T_{c}+V_{m}\right)}}{n e^{2} A}
$$

The Sc is destroyed, when $R$ is no longer imaginary, and this hold when:

$$
V_{m} \geq K T_{c}
$$

This means that the Sc is destroyed when the magnetic field strength exceeds a certain critical value corresponding to the critical magnetic potential:

$$
V_{m c}=K T_{c}
$$

which is in complete agreement with experiment.

But the magnetic potential $V_{m}$ is related to the magnetic flux density $B$ according to the relation

$$
V_{m}=\mu_{m} B \cos \theta
$$

where $\mu_{m}$ is the atomic dipole moment. Thus from (34) one gets

$$
B_{c}=K T_{c}\left(\mu_{m} \cos \theta\right)^{-1} n^{-1}
$$

where $n$ stands for the number of atoms having energy $E$. Thus according to Maxwell distribution law

$$
n=n_{o} \bar{e}^{x}\left(\mu_{m} \cos \theta\right)^{-1} n^{-1}
$$

where

$$
x=B E=\frac{E}{\bar{E}}=\frac{K T}{\gamma K T_{c}}=\frac{T}{T_{c}}
$$

where one uses Equation (17) and assumes the average energy to be given by

$$
\bar{E}=\gamma K T_{c}
$$

Thus for $\left(T<T_{c}\right), \quad x<1$

$$
n=n_{o}(1-x), n^{-1}=n_{o}^{-1}(1+x)
$$

Inserting (40) in (36) gives

$$
B_{c}=K T_{c}\left(\mu_{m} \cos e\right)^{-1}(1-x)(1+x)=K T_{c}\left(\mu_{m} \cos e\right)^{-1}\left(1-\gamma^{-2}\left(\frac{T}{T_{c}}\right)^{2}\right)
$$

which in the conventional relation of the critical in the stand and texts $[9,10]$. Where

$$
\gamma^{-2}=1,1.07
$$

\section{DISCUSSION}

Anew approach for expressing the wave function in the energy space is proposed here. In the conventional ordinary one the energy eigen function is spatial dependent as shown by Equation (8), and the energy wave function is time dependent, thus it gives the probability that the energy is $E_{k}$ at a certain time. In this new trend the energy eigen function is time dependent as Equation (7) indicates. Since $\Psi$, in 
Equation (5) depends on time and space, thus $c_{k}$ depend on $x$. Thus the energy wave function gives the probability, that the particle energy is $E_{k}$ at a certain point $x$.

Although (7) and (14) gives free travelling wave solution, but Equation (5) suggests that the wave function $\Psi$ is the form of a wave bucket as for as $K$ is changed, when the energy $E_{k}$ changes by changing the potential from point to point according to Equation (16). The energy wave function Equation (13) resembles that of Schrodinger.

By suggestion free travelling wave solution as suggested by Equation (14), a useful expression for the wave number is found in Equation (16). This wave number is energy and potential dependent. One can assume that the total energy in the form of thermal energy according to Equation (7), and assume repulsive electron cloud uniform potential $V_{0}$ to get a useful expression for the wave number in Equation (19).To relate the wave number to the resistance one utilizes the expression of it in terms of the momentum in classical mechanics and replace it by the quantum momentum which depends on the wave number $K$, as shown by Equation (21). The resistance is expressed in complex form according to Equation (20). The super conducting resistance vanishes when $R$ is a purely imaginary number. According to Equation (27) this happens when $T<T_{c}$

Which agrees completely with experimental observations.

The Sc is destroyed when an external magnetic field is applied which adds to the potential term an additional magnetic potential as shown by Equation (31). Thus the resistance $\mathrm{R}$ which is given by Equation (32), shows that the Sc is destroyed when the applied magnetic field exceeds a certain critical value according to Equation (34). The relation obtained for the critical magnetic field agrees with the empirical one.

This result agrees also with experimental observation which shows that Sc is destroyed when the applied external magnetic field exceeds a certain critical value $[9,10]$.

\section{CONCLUSION}

Using time dependent energy eigen function, the spatial evolution of the energy wave function is obtained. A useful expression for Sc resistance which vanishes beyond the critical temperature is found also. This expression conforms to that well known behavior of conventional superconductors. This model shows also that application of external magnetic field destroys superconductivity when it exceeds a certain critical value. A useful expression of the critical magnetic flux density in terms of the temperature and critical temperature which is typical to ordinary conventional one is also obtained.

\section{CONFLICTS OF INTEREST}

The authors declare no conflicts of interest regarding the publication of this paper.

\section{REFERENCES}

1. Annett, J.F. (2006) Superconductivity, Superfluid's and Condense States. OVP, Cambridge.

2. Poole Jr., C.P., Afrach, H. and Creswick, R.J. (2007) Superconductivity. Academic Press, London. https://doi.org/10.1016/B978-012088761-3/50034-X

3. Kumarsaxena, A. (2009) High Temperature Superconductors. Rewa, India. https://doi.org/10.1007/978-3-642-00712-5_2

4. Hammad, I.A.I., Dirar, M., Alatta, N.O., et al. (2017) Pressure Effect on Superconducting Critical Temperature According to String Model. International Journal of Fluid Mechanics \& Thermal Sciences, 3, 70-74.

https://doi.org/10.11648/j.ijfmts.20170306.12

5. Abdalrahman, R.I., Taha, R.A.E.M., Attia, I. and Abd-Alla, M.D. (2016) New Derivation of Simple Josephson Effect Relation Using New Quantum Mecjanical Equation. Natural Science, 8, 85-88. https://doi.org/10.4236/ns.2016.83011 
6. Allah, M.D.A., et al. (2006) Effect of Magnetic Field on Superconducting Complex Resistance According to Quantum Mechanics. Global Journal of Engineering Science and Researches, 3, 7.

7. Abdallah, M.D., Widaa, E.M.A. and Ahmed, S.A.E. (2015) Relation between Critical Temperature and Superconductivity Zero Resistance According to Quantum Laws. International Journal of Engineering Sciences \& Management, 5.

8. Zakaria, A., Altambori, A., Dirar, M., ELhussien, A., AbdAlgani, R. and Abdalla, A. (2016) Quantum Effect of Magnetic Field in Destroying Superconductivity. International Journal of Engineering Sciences \& Management.

9. Elliot, R.J. and Gibson, A.F. (1982) An Introduction to Solid State Physics. The MacMillan Press Ltd., London, Chapter 8, 343-350.

10. Burns, G. (1985) Solid State Physics. Academic Press, Orlando, Chapter 16, 650. 